\title{
sciendo
}

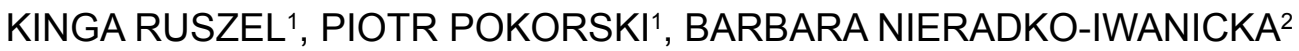

\section{Controversies about selenium supplementation}

\begin{abstract}
Introduction. Selenium (Se) is a trace element found mainly in meat, seafood, nuts and grains. Se is found in selenoproteins such as selenocystein or selenomethionin. A well balanced diet provides enough Se. Many regulatory and metabolic enzymes contain Se as their component, which is why Se supplementation is used in the treatment as well as prevention of multiple disorders. Se may, however, be toxic if overdosed.

Aim. The aim of this review is to summarize the data about functions of Se in human body and to discuss its use in treatment and prevention of diseases.

Materials and methods. The search was conducted using the PubMed and Google Scholar databases in March and April 2020. The key words used were: 'selenium', 'cardiovascular disease', 'selenium supplementation', 'Keshan disease', 'source of selenium'. A total of 68 articles were analysed.

Results. The first cases of chronic Se deficiency cases were documented 85 years ago in China. The patients with cardiomyopathy, extensive fibrosis and degenerative changes in the heart were diagnosed with Keshan disease. Human selenoproteonome consists of at least 25 selenoproteins. Se plays a role in immunity and metabolism via its role in functioning of numerous enzymes: glutathione peroxidase, thioredoxine and methionine sulfoxide reductase, methionine-sulfoxide reductase B1. Se plays a role in glucose homeostasis, Alzheimer's disease, thyroid disorders, infectious, inflammatory diseases, vascular diseases and fertility.

Conclusion. Se deficiency increases the risk of Keshan disease, but there is not enough evidence to recommend its supplementation for prevention of cardiovascular disease. However, Se status is important part of health assessment. Se supplementation should not exceed the dose of $55 \mu \mathrm{g} /$ day.
\end{abstract}

Keywords: selenium, selenoproteins, Keshan disease.

DOI: $10.2478 /$ pjph-2021-0005

\section{INTRODUCTION}

Selenium (Se) species (selenite or selenite) are prooxidants. Enzymes containing Se (for example glutathione peroxidase containing selenium-cysteine in their active site) are antioxidants. Se influences the inflammatory pathways responsible for modulation of the reactive oxygen species (ROS) by inhibiting the nuclear kappa B factor $(\mathrm{NF}-\mathrm{\kappa B})$ cascade. This inhibition stops the synthesis of interleukins and tumour necrosis actor alfa (TNF- $\alpha)$. Se regulates the migration, adhesion and the phagocytosis of leukocytes $[1,2]$. Se is also considered to be involved in the pathogenesis of circulatory disorders [3], it plays a role in glucose homeostasis [4], development of Alzheimer's disease [5], thyroid disorders [6], infectious [7] and inflammatory diseases [8], as well as fertility [9]. There are publications providing evidence that Se supplementation could be helpful in cancer prevention [10].

Se in human organisms comes from the diet, however both: Se excess or deficiency may cause dangerous consequences. Se intake is associated with the dwelling place and food choices. Many foods and dietary supplements contain Se in differ- ent chemical forms. In this group selenomethionine (Se-Met) is the compound with the highest Se bioavailability. Se status can be used as a prognostic factor. In patients suffering from systemic inflammatory response syndrome (SIRS), a decrease in Se concentration correlates with an increased mortality rate. In such situations patients are admitted to intensive care units (ICU) and intravenous Se supplementation is used for treatment [11]. However, physicians must keep it on mind that Se overdose can cause intoxication.

\section{AIM}

The aim of this review is to summarize the data involving the influence of Se as an antioxidant in the treatment of diseases.

\section{MATERIALS AND METHODS}

The search was conducted using the PubMed and Google Scholar databases in March and April 2020. The key words used during the research were: 'selenium', 'cardiovascular 
disease', 'selenium supplementation', 'Keshan disease', 'source of selenium'. A total of 68 articles published between 1988 and 2020 were analysed.

\section{RESULTS AND DISCUSSION}

Selenoproteins, found in our diets, contain Se. Current studies show that the human selenoproteonome consists of at least 25 selenoproteins. Selenoproteins are named with the prefix SELENO- and a letter: SELENOF, SELENOH, SELENOI, SELENOK, SELENOM, SELENON, SELENOO, SELENOP, SELENOS, SELENOT, SELENOV, SELENOW. This group of proteins include antioxidative enzymes such as: glutathione peroxidase (GPX), thioredoxine and methionine sulfoxide reductase [12]. The GPXs are thought to be the most important selenoproteins. Contrary to other antioxidants, GPX can neutralize ROS and reactive forms of nitrogen. They are involved in oxidising $\mathrm{H} 2 \mathrm{O} 2$ to water. In humans, the family of GPX enzymes consists of 6 enzymes involving Se: GPX1, GPX2, GPX3, GPX4, GPX5 and GPX6. Studies conducted on laboratory mice prove that GPX1 deficiency results in decreased protection from complications due to cardiac muscle damage, exacerbates ischemia-reperfusion injuries to cardiomyocytes, promotes endothelium dysfunction and structural irregularity of the cardiac muscle. Studies suggest that GPX3 is relevant in the proper functioning of the endothelium and the oxidation of low density lipoproteins (LDL) by the removal of soluble hydroxides, which is why GPX3 plays a role in preventing atherosclerosis $[13,14]$. GPX3 excess is also relevant, as it leads to myocardial hypertrophy [15]. GPX3 insufficiency leads to an increased platelet adhesion due to an increase in oxidative inactivation of NO. The impairment of physiological mechanisms inhibiting blood platelets promotes platelet hyperactivity leading to thrombosis [13]. GPX4 prevents LDL oxidation and can reduce hydroperoxides in endothelial cell membranes decreasing clotting [16]. Thioredoxin reductases (TXNRD1,2,3) are responsible for the regulation of many redox processes within a cell. TXNRDs are responsible for the signalisation, communication between cells, metabolism of the cell and repairing the deoxyrybonucleic acid (DNA). TXNRDs protect the cell form oxidative stress [17]. TXNRDs are a multifunctional proteins, capable of existing outside as well as inside the cell. Mitochondria are key structures in the regulation of cell apoptosis, every change in the redox environment within the cell leads to the exhaustion of reduced forms of nicotinamide adenine dinucleotide phosphate (NADP) and glutathione. Mitochondria have multiple protective antioxidants, however TXNRD2 plays the main role. Many different illnesses such as diabetes, ischaemic heart disease, acquired immunodeficiency syndrome (AIDS), hypertension may lead to changes in TXNRD concentration [18]. TXNRD2 is present in multiple tissues in the human body, its' highest concentrations are found in highly metabolically active tissues including: ovaries, liver, heart, adrenal glands and neurons [19]. TXNRDs are activated by oxidative stress. Endothelial cells irritated with $\mathrm{H} 2 \mathrm{O} 2$ increase the expression of TXNRDs which promotes the growth of lymphocyte, fibroblast populations and multiple lines of cancer cells, which is why TXNRDs are also considered a growth factor [18-20].

Methionine-sulfoxide reductase B1 (MSRB1) and methionine-sulfoxide reductase A (MSRA) belong to the group of methionine sulfoxide reductases. MSRs are responsible for reducing oxidised methionine bound to protein and other molecules. Studies show that a decrease in SeIR concentration leads to a decreased cell tolerance for oxidative stress. SeIR is mainly expressed in the cytoplasm and the nuclei of cells, being involved in reducing the oxidative stress in cardiomyocytes, it is thought to explain its role in the regulation of cardiac muscle hypertrophy and the reperfusion of ischaemic hearts in mice models [21]. SeIR plays an important role in aging mechanisms and neurological degeneration. Bacteria and yeast cells without the MSR gene show decreased tolerance or oxidative stress and shorter lifespans [22]. The overexpression of the MSRA in human T lymphocytes extends their lifespans in oxidative stress conditions. MSR may have three important functions in the human body: as an antioxidant enzyme, repair enzyme and as a regulatory enzyme [23]. The MSRA is highly expressed in retinal epithelial cells, macrophages, liver, kidneys, cerebellum and brain neurons [24]. A decrease in SeIR concentration may lead to the loss of protection from oxidative stress, increased oxidative damage to cells and a shorter cell lifespan. The decrease in MSRA activity can be caused not by declining concentration of the enzyme but rather the structural modification of the enzyme. This hypothesis was confirmed in studies comparing SeIR activity in the mitochondria and the cytoplasm from cardiomyocytes isolated from left ventricles of rat hearts exposed to different periods of ischemia and reperfusion. These studies included 3 stages:0-90 min perfusion, $30 \mathrm{~min}$ occlusion of the anterior interventricular branch of left coronary artery and finally $30,60,90$ or $120 \mathrm{~min}$ of reperfusion. After the above mentioned stages of the experiment, SeIR activity was determined in the mitochondria and the cytoplasm using Western blot. There was no change in the concentration of MSRA during ischemia and reperfusion, indicating that MSRA protein was not degraded [21].

Selenoprotein K (SelK) is another selenoprotein, involved in protection of atherosclerosis development and progression. Examination of the expression pattern revealed that the human SelK messenger ribonucleic acid (mRNA) has been highly present in the cardiac muscle. It was later found that overexpression of SelK decreases intracellular ROS concentration which protects cardiomyocytes from the toxic effects off oxidative stress [25].

The SELENOS is an antioxidative protein resistant to the endoplasmic reticulum. This selenoprotein is relevant in disorders involving the metabolism, immune system and inflammatory reaction. It is highly expressed within skeletal muscle and plays an important role in muscle growth and development. Selenoproteins are becoming increasingly important in proper muscle contraction $[26,27]$. The degradation of $50-75 \%$ of SELENOS1 in myoblasts resulted in an increased susceptibility to oxidative stress, decreased proliferation and life span off cells, higher concentration of $\mathrm{H} 2 \mathrm{O} 2$, decreased reduced glutathione (GSH), glutathione disulfide-oxidized (GSSG) and an increased expression of endoplasmic reticulum genes and oxidative stress markers (26). The SELENOS1 is responsible for regulating contraction and reaction to oxidative stress in quickly contracting muscles [28].

Similar to other proteins, SELENOS contains Se in the form of selenocysteine and is involved in redox reactions in the human body. Mutations to the SELENON1 gene may result in the development of SELENON1 related myopathy (SELENONRM) which manifests itself with muscle weakness, stiffness of the spine and respiratory failure. SELENON insufficiency 
leads to irregular development of the lungs manifested as enlarged alveoli. The histopathological examination revealed changes ranging from slightly dystrophic to inherited myopathy with myofibril disorganisation [29,30]. Different types of inherited muscle dystrophies present themselves with early muscle hypotonia especially of the axial muscles. A study by Deniziak about ultrastructural analysis of myotomes revealed defective sarcomere organisation in the muscle and an altered integrity of muscle tissue, pointing to the high relevance of SELENON in muscle development. Mutations in the SELENON1 gene lead to irregular muscle tissue architecture [27]. A magnetic resonance imaging (MRI) scan should be conducted in case that myopathy is suspected. An MRI scan of lower extremities revealed diffuse lipid infiltration and hypotrophy of the sartorius, great adductor and semimembranosus muscles in one of the patients, while a different patient suffered only from the isolated hypotrophy of the sartorius muscle [30]. Data from a study conducted on 41 patients aged from 1 to 60 years suffering from SELENON-RM showed that the average age when patients need non-invasive mechanic ventilation is 13.9 years of age and the average age of scoliosis detection is 10 years. Respiratory failure usually develops around the age of 14 years, however the first symptoms may appear during infancy or later in the 4th decade of life [31]. Information about selenoproteins is summarized in Figure 1.

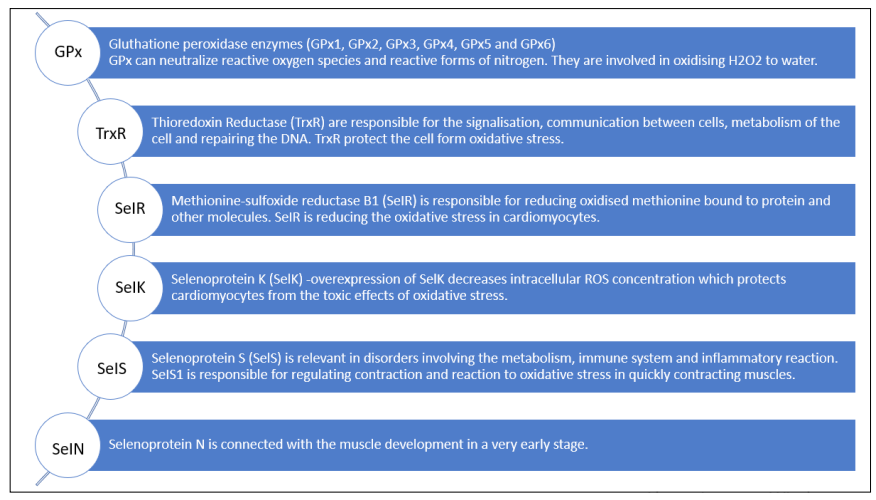

FIGURE 1. Selenoproteins in human body.

The potential role of Se in the development and progress of circulatory disorders has been studied for decades. The first cases of chronic Se deficiency were documented 85 years ago in China. The patients described were diagnosed with progressing cardiomyopathy, including extensive fibrosis and degenerative changes in the heart muscle, known as Keshan syndrome [32]. Samples of cardiac tissue from diseased patients who suffered from Keshan disease appeared pale due to extensive fibrosis and necrosis, furthermore the state of sarcolemic architecture of the muscle suggested myolysis [3335]. Studies revealed that despite Se supplementation in the endemic region, new cases of chronic Keshan disease were identified. Recent research shows that Coxsackie B3 virus may be a potential factor contributing to the development of Keshan disease too. Administration of Se supplements may reduce platelet reactivity and lower the risk of perivascular necrosis of the cardiac muscle and lower the risk of acute Keshan disease. The relationship between low Se concentration in rice and the endemic occurrence of Keshan disease was noticed in 1970. The studies conducted back in the 70's became the foundation for the Chinese Ministry of Health to engineer a Keshan disease prevention strategy. The prevention strategy was based on Se supplementation involving supplement tablets and $\mathrm{Se}$ enriched salt administered to the people inhabiting regions endemic to Keshan disease [36]. The strategy has practically eliminated Keshan disease in the endemic area [34].

The meta-analysis of previously gathered data shows a moderate and statistically significant correlation between the concentration of Se and the results of coronary disease progression in patients. A $50 \%$ increase in Se concentration in the blood resulted in a $24 \%$ decrease of development of the coronary heart disease. The observational study was not able to determine the effect of Se alone and differentiate it from the effect of other antioxidants and vitamins, including $\beta$-carotene, and vitamin $\mathrm{E}$ on the cardiovascular system [37]. Low levels of Se in the blood may lead to: an increased risk of cardiovascular disease by intensifying the synthesis of thromboxane from prostaglandin, increased platelet aggregation and vasoconstriction [38]. By binding to toxic metals and forming nonreactive compounds, Se may protect the cardiovascular system from the effects of metals involved in the pathogenesis of atherosclerosis, preventing the aggravation of coronary disease. In Taiwan, a group of patients suffering from the Blackfoot disease, causing gangrene of lower extremities, a low concentration of Se was determined. All patients suffering from Blackfoot had an increased concentration of lactate dehydrogenase (LDH) which was hypothesised to be connected to muscle gangrene. Selenium supplementation could be beneficial for the patients with Se deficiency suffering from Blackfoot [38]. There is a positive correlation between Se blood levels and hypertension, irrespectively of the patients age and medication prescribed. National Health and Nutrition Survey (NHANES) conducted an analysis of 6683 cases between 2011 and 2016 concerning the role of trace elements in the pathogenesis of hypertension in age groups $8-80$ years of age $[39,40]$. Similar study concerning Se intake in the diets of 2638 NHANES participants has been conducted in 2003-2004 and revealed the same positive correlation between the increase in Se concentration in the blood and the increase in blood pressure $[41,42]$. Despite multiple publications confirming the beneficial effects of Se on prevention of coronary disease, no recommendations for this element intake are being made for patients, mainly due to the difficulties in interpreting observational studies. Furthermore, Se has commonly been associated with other vitamins and minerals in commercial formulas and food products making it impossible to extract particular effects of Se.

Zhang et al. in their meta-analysis of 16 studies concerning the role of Se in pathogenesis of cardiovascular disease indicate the importance of considering Se status in health assessment regarding Se supplementation as intervention aiming at prevent the diseases. The meta-analysis revealed a nonlinear relationship between cardiovascular disease with blood $\mathrm{Se}$ concentrations of $30-165 \mu \mathrm{g} / \mathrm{l}$. Authors noticed the benefit of cardiovascular disease within Se range 55-145 $\mu \mathrm{g} / \mathrm{l}$. They reported that Se supplementation $(200 \mu \mathrm{g} /$ day $)$ for 2576 raised the blood Se concentrations by $56.4 \mu \mathrm{g} / 1$ but supplementation with $100 \mu \mathrm{g}$ /day for 24-456 weeks had no effect on cardiovascular disease [43]. According to the present knowledge, SELENOP is a reliable marker of body selenium status [44].

$\mathrm{Se}$ is sometimes referred to as a two-faced element due to some of its toxic and beneficial effects. The range of healthy Se levels is very narrow. The WHO states that the toxic limit of Se intake is $800 \mu \mathrm{g}$ daily [45]. A study investigating 201 
patients with Se poisoning described wide variety of symptoms of Se poisoning. While the recommended daily intake of Se is $55 \mu \mathrm{g} / \mathrm{d}$ and the highest acceptable daily intake is 400 $\mu \mathrm{g} / \mathrm{d}$, the patients suffering from Se poisoning took on average $41749 \mu \mathrm{g} / \mathrm{d}$ of Se. The symptoms of Se poisoning included: diarrhoea $(78 \%)$, fatigue $(75 \%)$, hair loss $(72 \%)$, joint pain (70\%), discoloration or brittleness of nails (61\%), bad mouth odour and nausea $(58 \%)$. Selenium toxicity was endemic in some parts of China due to very high concentration of that element in the soil [45]. Se toxicity is currently rare, due to accidental overdose or suicidal attempt [32].

However, according to the recent publication of Rayman, some people can easily tolerate low or high levels of Se. The author suggests that it depends on polymorphisms in a gene, which can improve one's chances to tolerate low or high Se level [44]. Moreover, the same scientist showed data confirming that Se can interact with toxic elements in human body and form safer complexes. The same author discusses dual nature of certain selenoproteins. For instance, selenomethionine can be toxic, at high concentrations leading to overproduction of superoxide radicals reacting with thiols or diselenides to produce selenyl sulphides. Interestingly, high consumption of Se was found to affect the gut microbiota and thanks to that, Se could be faster excreted and its toxicity is reduced $[44,46]$.

Epidemiological studies have linked an increased risk of autoimmune thyroiditis, Graves' disease and goitre to low selenium status. Trials of selenium supplementation in patients with chronic autoimmune thyroiditis have generally resulted in reduced thyroid autoantibody titre without apparent improvements in the clinical course of the disease. In Graves' disease, selenium supplementation might lead to faster remission of hyperthyroidism and improved quality of life and eye involvement in patients with mild thyroid eye disease. Despite recommendations only extending to patients with Graves ophthalmopathy, selenium supplementation is widely used by clinicians for other thyroid phenotypes. Ongoing and future trials might help identify individuals who can benefit from selenium supplementation, based, for instance, on individual selenium status or genetic profile [6].

There are publications about the influence of Se status on glucose homeostasis. Negative correlation between blood Se level and glycosylated hemoglobin was observed in patients with type 1 diabetes in the study by Ruiz [47].

The role of Se in pathogenesis of type 2 diabetes was discussed in the study by Ogawa et al. [48]. Authors underlined that Se supplementation is beneficial for people with deficiency of that element, but supplementation with Se may increase the risk of type 2 diabetes in males. Authors state that the connection between Se level and glucose level is sexually dimorphic [48].

Jablonska et al. evaluated the influence of Se supplementation on expression of genes, which are involved in synthesis of molecules involved in glucose homeostasis. They used cDNA from 76 patients without diabetes who received Se supplements at the dose of $200 \mu \mathrm{g}$ for 6 weeks. Authors collected blood from subjects before supplementation, after 2 weeks of Se dosing, after 4 weeks, and finally 4 weeks after washout. Fifteen genes were selected (encoding proteins that are involved in insulin signaling and metabolism of glucose). Glycosylated hemoglobin and fasting plasma glucose were measured 4 times during the course of the study. Supplementation of Se was associated with a decrease in the level of glyco- sylated hemoglobin, but not with fasting plasma glucose level. In the study significant down-regulation of several genes encoding proteins involved in glucose homeostasis was observed. It suggests that Se affects blood glucose level regulation related to insulin production, glycolysis, metabolism of pyruvate [49].

Because many studies showed inconsistent relationship between Se intake and the risk of cardiovascular diseases and type 2 diabetes, Rees et al. conduced a systematic review of twelve clinical trials and found no significant correlation between Se supplementation and mortality (neither of all causes, nor of cardiovascular diseases). Interestingly, there was slightly increased risk of type 2 diabetes in people taking Se supplements but without statistical significance [46].

Se has an influence on gestational diabetes. A study with 60 participants suffering from gestational diabetes was conducted. Part of them was randomly selected to receive $100 \mu \mathrm{g}$ of Se supplementation daily for 12 weeks beginning from the 24-28th week of gestation. The study revealed that glucose concentration in urine, blood glucose levels 2 hours post meal, glycosylated haemoglobin, plasma insulin levels and homeostatic model assessment (HOMA IR) did not differ significantly between the group receiving Se supplementation and the group receiving placebo. The study suggested that $100 \mu \mathrm{g}$ of Se supplementation daily did not affect glucose homeostasis in patients suffering from gestational diabetes [4].

Acting as antioxidants, Se containing compounds plays a role in the functioning of the immune system. Se is found in high concentrations in lymphatic cells of the liver, spleen and lymph nodes [50]. Due to mixed opinions on whether Se influences the immunological responses in a cell, a study investigating the effect of $\mathrm{Se}$ on the proliferation of $\mathrm{T}$ cells and the synthesis of interleukin 2 (IL-2) in pig spleens was conducted. The $\mathrm{T}$ cells were stimulated with mitosis inducing factors in the presence of sodium selenite $(0.5-4 \mu \mathrm{mol} / \mathrm{L})$. The study revealed that Se increased the expression of T cell receptors (TCR), proliferation of T cells and the synthesis of IL-2 by a statistically significant margin. Additionally, Se increased the concentration of GPX1 and the mRNA of thioredoxin reductase, increased the activity of GPX1 and the concentration of reduced glutathione in splenocytes [51]. Another study conducted on dairy cows revealed that selenium could aid the immune system in mitigating oxidative stress and decrease the intensity of inflammatory disorders affecting the production efficiency of dairy cows such as mastitis or uterine inflammation [52]. Many of the health benefits of Se can be attributed to the antioxidative properties of seleoproteins. A change in selenoprotein activity can directly influence key reactions and functions in cells engaged in the inflammatory response. Addition of Se to neutrophils in vitro successfully improved the chemotactic migration and increased the synthesis of peroxide necessary for eradicating bacteria and other pathogens. The study found that in comparison to lymphocytes of dairy cows with adequate selenium levels, the lymphocytes isolated from the blood of the dairy cows affected by selenium deficiency, had a lower mitogen induced proliferation rate and a decreased biosynthesis of eicosanoids (5-lipoxygenase pathway) [5254]. It is hypothesised that Se deficiency increases the synthesis of platelet activating factor (PAF) in human endothelial cells, however the mechanism remains unclear. According to a different study on dairy cows, endothelial cells of the aorta with insufficient Se levels, when stimulated by TNF $\alpha$ would 
synthesise significantly more platelet activating factor (PAF) than the cells with adequate Se concentration. Se deficiency changes the rate of PAF synthesis by altering the activity of anabolic enzymes, downregulating the synthesis of prostacyclin's [55].

Gombart et al. analyzed the rose of Se in the course of infectious diseases and found that selenoproteins are important for antioxidant host defense system, affecting leukocyte and natural killer (NK) cell function, help increase interferon $\gamma$ production. Se helps to maintain antibody levels. According to Gombart et al. Se deficiency causes suppression of immune function, diminished NK-cell cytotoxicity, impaired humoral and cell-mediated immunity, decreased immunoglobulin titers, impaired cell-mediated immunity, increased viral virulence and decreased response to vaccination [7]. Authors emphasize that Se supplementation improves cell-mediated immunity, improves $\mathrm{T}$ helper cell counts, enhances immune response to viruses in deficient individuals [7].

There are also studies analyzing the role of Se in pathogenesis of Alzheimer's disease (AD. Selenoprotein P (SELENOP), the main Se transport protein, is responsible for maintaining Se homeostasis in the body. SELENOP is necessary for proper brain development and functioning. Authors conclude that via redox reactions Se deficiency may play a role in $\mathrm{AD}$ development [8].

Stroke is a result of brain blood vessel disease. Studies were conducted to find correlation between Se status and the risk of stroke. In the years 2007-2011 data from 7065 Canadians and in the years 2011-2012 from 5030 residents of the United States (US) were analyzed in this aspect. A total of 284 strokes were identified in both groups together. It was found that subjects with stroke had lower Se levels if compared to those without stroke. Decreasing risk of stroke was observed with blood Se levels rising but the curve plateaued at $190 \mu \mathrm{g} / \mathrm{L}$. Authors concluded that there is an inverse association between blood Se level and the risk of stroke in the US and Canadian citizens [56].

There is data that Se- containing compounds modulate inflammation and Se supplementation helps in resolution of inflammation. That is thanks to bioactive lipid mediators and prostanoids. These lipid mediators enable interaction of immune cells, while cellular redox gatekeeper -selenoproteins further aid to reduce inflammation [8].

Se is also believed to play a role in male and female infertility. As oxidative stress can seriously impair male, and possibly also female, antioxidant properties of Se compounds is considered beneficial for fertility. There are indications that Se influences growth, maturation, replication of oocytes. Authors suggest that Se supplementation at the dose less than $200 \mu \mathrm{g} / \mathrm{d}$ is beneficial for men and improves sperm motility [9].

The antitumour properties of Se remain poorly understood. Possible antineoplastic activity of Se depends on the chemical form of the element, its' dose and the type of tumour. Studies show that the Se supplementation of yeasts decreases the morbidity of prostate cancer by $60 \%$ and a $50 \%$ decrease in total mortality rates in both lung cancer and colorectal cancer. High doses of Se increase the synthesis of oxygen free radicals, which shows to have a beneficial antineoplastic effect. Oxygen free radicals initiate apoptosis playing an active role in alternating the conformation of proteins, such as: transcription factors, signalling proteins and supressing enzymes, all of which are necessary for the survival of the cell $[10,57,58]$. A series of experiments conducted on animal subjects determined that $\mathrm{Se}$ supplementation added to the animal diet, decreased carcinogenesis in the group of animals exposed to carcinogens [38].

People should obtain Se from regular diet. Animal and plant tissues contain trace amounts of this element. The largest amounts of Se can be found in the Earth's crust, which makes plant foods and water good sources of this element for humans. The main source of Se found in soil is the erosion of rocks containing selenites and selenides. Selenite and selenide are commonly found in soil around the world, however their concentrations vary depending on the amount of organic matter in and around the soil, the amount of rainfall and the soils texture type. Inorganic Se compounds are found in water, while organic Se compounds (selenomethionine, selenocysteine) are mainly found in grain and vegetables. The intake of selenium by vegetables depends on physical and chemical factors, such as: $\mathrm{pH}$, redox state and microbiological activity. Soil acidity determines the rate at which Se is absorbed by plants from the soil. Alkaline soil makes the absorption easier [59]. Selenite is more mobile and bioavailable than other forms of Se and is the most often accumulated form of Se found in plants [60]. The average Se content in cultivated soil ranges between 0.33 and $2 \mathrm{mg} / \mathrm{kg}$ across the world. Se rich soils are found in the US, Russia and parts of China, however majority of the soils in China contain very small amounts of selenium $(<0.1 \mathrm{mg} / \mathrm{kg})$ [57]. Soils which arose form Se rich rocks such as sandstone and limestone have a high level of this element [59]. Low levels of Se may have a beneficial effect on a plant growth rate, making it grow faster, protecting from abiotic stress factors. High levels of Se in the soil may stop plants growth and interfere with plant metabolism. The beneficial or negative effect of Se on plant growth rate depends on the type of plant, Se soil content and the compound of Se [60].

Water contains trace amounts of Se. Main forms of Se detectable in water are selenines and seleniates [61]. Underground water has a much higher Se concentration than seawater [62]. According to the World Health Organisation (WHO), the permissible amount of Se in drinking water is $10 \mu \mathrm{g} / \mathrm{L}$ [57].

Se concentration in plants is not connected with the concentration of this element in the surrounding soil. Some plants actively accumulate Se at concentrations that far exceed those in the soil. Grasses usually contain higher concentrations of Se than legumes, however this difference in concentration becomes less significant in soils with a low Se content. Cereals can store Se in their seeds mainly in the form of selenomethionin. The amount of selenomethionin in seeds differs depending on the region [50].

There is a constant biochemical exchange of Se compounds in the atmosphere. The presence of this element in the air is caused by soil erosion, forest fires, volcanic explosions and human activity such as pollution and the burning of fossil fuels. The content of Se in the air is low and ranges from 1 to $10 \mathrm{ng} / \mathrm{m} 3$ [63].

Se content in foods depends on the geographic region, season, changes in weather conditions and the degree of food processing, which demands for occasional testing of Se levels found in soil and food. The main source of Se for humans is the dietary intake [64]. Se deficiency is an indication for supplementation. There is an increasing amount of new generation of Se supplements with a low risk of overdose and intoxication [65]. New methods of growing food are also being introduced to increase Se content. Different forms of Se ap- 
plication were used for the two varieties of 'Golden Delicious' and 'Jonagold' apples grown in 2017 and 2018 to increase its' absorption. Higher fertilizer levels resulted in higher Selenium accumulation in the fruits and higher intake of the element in people consuming a typical western diet [66].

There is an interesting publication of Schomburg who states that positive health effects of supplemental Se can be interpreted as the consequence of correcting deficiency [67]. According to the author, Se supplementation should be given not only to well defined patients with thyroid gland diseases in order to alleviate symptoms, improve the disease and help to cure it as adjuvant, but also after nutritional assessment, correcting deficiency to patients without diagnosed thyroid pathology. Author underlines that in Se deficiency, the lack of this element causes limitation for the required adaptation of selenoprotein expression. At the same time Schomburg emphasized that supplemental Se does not cause diabetes [67]. Rayman also states that Se status assessment is essential for supplementation decision [68]. As both, Se deficiency and excess, are found throughout the world, Se excess can develop due to supplementation with Se. Both these conditions have been associated with negative health effects that often are characterized by a U-shaped relationship. Increased mortality may result from low Se level as well as from too high level of this element in the blood. Some people can tolerate high Se level thanks to polymorphisms in genes improving body's ability to cope with high Se. In certain populations exposed to toxic elements, thanks to interaction with Se, safe complexes form. Positive or negative effects of Se supplementation depend on the type of formula used. At high dose selenomethionine has toxic effects. It is mediated by metabolism to selenols or selenolates, that generates superoxide radicals reacting with thiols and diselenides to produce selenyl sulphides or disulphides. Rayman also underlines that subjects exposed to high Se dosed from birth can alter their gut microbiota and thanks to that the excessive amount of Se is excreted [68].

According to Nutrition Standards for the Polish Population of 2017, the average selenium demand in the infant group is $15 \mu \mathrm{g}$ /day. For children aged 1-3 years $17 \mu \mathrm{g}, 4-9$ years $23 \mu \mathrm{g}$. For boys and girls aged 10-12 years $35 \mu \mathrm{g}$. For boys and girls aged 13-18 years it is $45 \mu \mathrm{g}$. Similarly for adult men and women. During pregnancy, the selenium requirement increases to $50 \mu \mathrm{g} /$ day. It increases to $60 \mu \mathrm{g}$ a day during lactation [69].

\section{CONCLUSIONS}

Se plays a role in immunity and metabolism via its role in functioning of numerous enzymes. It plays a role in glucose homeostasis, Alzheimer's disease, thyroid disorders, infectious and inflammatory diseases, vascular diseases and fertility. Se deficiency increases the risk of Keshan disease, but there is not enough evidence to recommend its supplementation for prevention of cardiovascular disease. However, Se status is important part of health assessment. Se supplementation should not exceed the dose of $55 \mu \mathrm{g} /$ day.

\section{REFERENCES}

1. Steinbrenner H, Al-Quraishy S, Dkhil MA, et al. Dietary selenium in adjuvant therapy of viral and bacterial infections. Adv Nutr. 2015;6(1):73-82.

2. Yang L, Wang W, Chen J, Wang N, Zheng G. A comparative study of resveratrol and resveratrol-functional selenium nanoparticles: Inhibiting amyloid $\beta$ aggregation and reactive oxygen species formation properties. J Biomed Mater Res A. 2018;106(12):3034-41.

3. Ingles DP, Cruz Rodriguez JB, Garcia H. Supplemental vitamins and minerals for cardiovascular disease prevention and treatment. Curr Cardiol Rep. 2020;22(4):22.

4. Sadat Najib F, Poordast T, Rezvan Nia M, Hossein Dabbaghmanesh M. Effects of selenium supplementation on glucose homeostasis in women with gestational diabetes mellitus: A randomized, controlled trial. Int J Reprod Biomed (Yazd). 2020;18(1):57-64.

5. Solovyev N. Selenoprotein P and its potential role in Alzheimer's disease. Hormones (Athens). 2020;19(1):73-9.

6. Winther KH, Rayman MP, Bonnema SJ, Hegedüs L. Selenium in thyroid disorders - essential knowledge for clinicians. Nat Rev Endocrinol. 2020;16:165-76.

7. Gombart AF, Pierre A, Maggini S. A review of micronutrients and the immune system - working in harmony to reduce the risk of infection. Nutrients. 2020;12(1):236.

8. Qian F, Misra S, Prabhu KS. Selenium and selenoproteins in prostanoid metabolism and immunity. Crit Rev Biochem Mol Biol. 2019;54 (6):484-516.

9. Mintziori G, Mousiolis A, Duntas LH, Goulis DG. Evidence for a manifold role of selenium in infertility. Hormones (Athens). 2020;19(1):55-9.

10. Raganová A, Gažová A, Tomo I, Kristová V. Selenium in the prevention and subsidiary therapy of cancer of soft tissues. Ceska Slov Farm. 2018;67(2):66-70.

11. Hargreaves IP, Mantle D. Supplementation with selenium and coenzyme Q10 in critically ill patients. Br J Hosp Med (Lond). 2019;80(10):589-93.

12. Gladyshev VN, Arnér ES, Berry MJ, et al. Selenoprotein gene nomenclature. J Biol Chem. 2016;291(46):24036-40.

13. Barale C, Cavalot F, Frascaroli C, et al. Association between high on-aspirin platelet reactivity and reduced superoxide dismutase activity in patients affected by Type 2 Diabetes Mellitus or primary hypercholesterolemia. Int J Mol Sci. 2020;21(14):4983.

14. Forgione MA, Cap A, Liao R, et al. Heterozygous cellular glutathione peroxidase deficiency in the mouse - Abnormalities in vascular and cardiac function and structure. Circulation. 2020;106(9):1154-8.

15. Huang JQ, Ren FZ, Jiang YY, et al. Selenoproteins protect against avian nutritional muscular dystrophy by metabolizing peroxides and regulating redox/apoptotic signaling. Free Radic Biol Med. 2015;83:129-38.

16. Rose AH, Hoffmann PR. Part - selenoproteins and cardiovascular stress Thromb Haemost. 2015;113(3):494-504.

17. Lu J, Holmgren A. The thioredoxin antioxidant system. Free Radic Biol Med. 2014;66:75-87.

18. Chong CR, Chan WP, Nguyen TH, et al. Thioredoxin - interacting protein: Pathophysiology and emerging pharmacotherapeutics in cardiovascular disease and diabetes. Cardiovasc Drugs Ther. 2014;28(4):347-60.

19. Scalcon V, Bindoli A, Rigobello MP. Significance of the mitochondrial thioredoxin reductase in cancer cells: An update on role, targets and inhibitors. Free Radic Biol Med. 2018;127:62-79.

20. Iqbal MA, Eftekharpour E. Regulatory role of redox balance in determination of neural precursor cell fate. Stem Cells Int. 2017;2017:9209127.

21. Razavi AC, Bazzano LA, He J, et al. Novel findings from a metabolomics study of left ventricular diastolic function: The Bogalusa Heart Study. J Am Heart Assoc. 2020;9(3):e015118.

22. Jiang B, Adams Z, Moonah S, et al The antioxidant enzyme methionine sulfoxide reductase A (MsrA) interacts with Jab1/CSN5 and regulates its function. Antioxidants (Basel). 2020;9(5):452.

23. Méndez AA, Pena LB, Benavides MP, Gallego SM. Priming with NO controls redox state and prevents cadmium-induced general up-regulation of methionine sulfoxide reductase gene family in Arabidopsis. Biochimie. 2016;131:128-36.

24. Lourenço Dos Santos S, Petropoulos I, Friguet B. The oxidized protein repair enzymes methionine sulfoxide reductases and their roles in protecting against oxidative stress, in ageing and in regulating protein function. Antioxidants (Basel). 2018;7(12):191.

25. Pitts MW, Hoffmann PR. Endoplasmic reticulum-resident selenoproteins as regulators of calcium signaling and homeostasis. Cell Calcium. 2018;70:76-86.

26. Addinsall AB, Martin SD, Collier F, et al. Differential regulation of cellular stress responses by the endoplasmic reticulum- resident Selenoprotein $\mathrm{S}$ (Seps1) in proliferating myoblasts versus myotubes. Physiol Rep. 2018;6(24). 
27. Prajapati RS, Mitter R, Vezzaro A, Ish-Horowicz D. Greb1 is required for axial elongation and segmentation in vertebrate embryos. Biol Open. 2020;9(2):bio047290.

28. Addinsall AB, Wright CR, Shaw CS, et al. Deficiency of selenoprotein S, an endoplasmic reticulum resident oxidoreductase, impairs the contractile function of fast-twitch hindlimb muscles. Am J Physiol Regul Integr Comp Physiol. 2018;315(2):380-96.

29. Moghadaszadeh B, Rider BE, Lawlor MW, et al. Selenoprotein N deficiency in mice is associated with abnormal lung development. FASEB J. 2013;27(4):1585-99.

30. Ardissone A, Bragato C, Blasevich F, et al. SEPN1-related myopathy in three patients: novel mutations and diagnostic clues. Eur J Pediatr. 2016;175(8):1113-8.

31. Scoto M, Cirak S, Mein R, et al SEPN1-related myopathies: clinical course in a large cohort of patients. Neurology. 2011;76(24):2073-8.

32. Benstoem C, Goetzenich A, Kraemer S, et al. Selenium and its supplementation in cardiovascular disease - What do we know? Nutrients. 2015;7(5):3094-118.

33. Zhou H, Wang T, Li Q, Li D. Prevention of Keshan disease by selenium supplementation: A systematic review and meta-analysis. Biol Trace Elem Res. 2018;186(1):98-105.

34. Loscalzo J. Keshan disease, selenium deficiency, and the selenoproteome. N Engl J Med. 2014;370(18):1756-60.

35. Gaaloul I, Riabi S, Harrath R, et al. Coxsackievirus B detection in cases of myocarditis, myopericarditis, pericarditis and dilated cardiomyopathy in hospitalized patients. Mol Med Rep. 2014;10(6):2811-8.

36. Chen J. An original discovery: Selenium deficiency and Keshan disease (an endemic heart disease). Asia Pac J Clin Nutr. 2012;21(3):320-6.

37. Ju W, Li X, Li Z, et al. The effect of selenium supplementation on coronary heart disease: A systematic review and meta-analysis of randomized controlled trials. J Trace Elem Med Biol. 2017;44:8-16.

38. Roman M, Jitaru P, Barbante C. Selenium biochemistry and its role for human health. Metallomics. 2014;6(1):25-54.

39. Lin SM, Yang MH. Arsenic, selenium, and zinc in patients with blackfoot disease. Biol Trace Elem Res. 1998;15:9.

40. Bastola MM, Locatis C, Maisiak R, Fontelo P. Selenium, copper, zinc and hypertension: an analysis of the National Health and Nutrition Examination Survey (2011-2016). BMC Cardiovasc Disord. 2020;20. [https:// www.ncbi.nlm.nih.gov/pmc/articles/PMC6995060.]

41. Su L, Jin Y, Unverzagt FW, et al. Longitudinal association between selenium levels and hypertension in a rural elderly Chinese cohort. J Nutr Health Aging. 2016;20(10):983-8.

42. Kuruppu D, Hendrie HC, Yang L, Gao S. Selenium levels and hypertension: a systematic review of the literature. Public Health Nutr. 2014;17(6):1342-52.

43. Zhang X, Liu C, Guo J, Song Y. Selenium status and cardiovascular diseases: meta-analysis of prospective observational studies and randomized controlled trials. Eur J Clin Nutr. 2016;70(2):162-9.

44. Rayman MP. Selenium intake, status, and health: a complex relationship. Hormones (Athens). 2019;19(1):9-14.

45. Reich HJ, Hondal RJ. Why Nature Chose Selenium. ACS Chem Biol. 2016;11(4):821-841.

46. Rees K, Hartley L, Day C, et al. Selenium supplementation for the primary prevention of cardiovascular disease. Cochrane Database Syst Rev. 2013;(1):CD009671.

47. Vinceti M, Filippini T, Rothman KJ. Selenium exposure and the risk of type 2 diabetes: a systematic review and meta-analysis. Eur J Epidemiol. 2018;33(9):789-810.

48. Ogawa-Wong AN, Berry MJ, Seale LA. Selenium and metabolic disorders: An emphasis on type 2 diabetes risk. Nutrients. 2016 6;8(2):80.

49. Jablonska E, Reszka E, Gromadzinska J, et al. The effect of selenium supplementation on glucose homeostasis and the expression of genes related to glucose metabolism. Nutrients. 2016;8(12): E772.

50. Mehdi Y, Hornick J-L, Istasse L, Dufrasne I. Selenium in the environment, metabolism and involvement in body functions. Molecules. 2013;18(3):3292-311.

51. Ren F, Chen X, Hesketh J, et al. Selenium promotes T-Cell response to TCR-stimulation and ConA, but not PHA in primary porcine splenocytes. PLoS One. 2012;7(4). [https://www.ncbi.nlm.nih.gov/pmc/articles/ PMC3328446.]

52. Sordillo LM. Selenium-dependent regulation of oxidative stress and immunity in periparturient dairy cattle. Vet Med Int. 2013. [https://www.ncbi. nlm.nih.gov/pmc/articles/PMC3557619.]

53. Mattmiller SA, Carlson BA, Sordillo LM. Regulation of inflammation by selenium and selenoproteins: impact on eicosanoid biosynthesis. J Nutr Sci. 2013;2:e28.
54. Lopreiato V, Mezzetti M, Cattaneo L, et al. Role of nutraceuticals during the transition period of dairy cows: a review. J Anim Sci Biotechnol. 2020;11:96.

55. Sordillo LM. Selenium-dependent regulation of oxidative stress and immunity in periparturient dairy cattle. Vet Med Int. 2013;2013:154045.

56. Hu XF, Stranges S, Chan LHM. Circulating selenium concentration is inversely associated with the prevalence of stroke: Results from the Canadian Health Measures Survey and the National Health and Nutrition Examination Survey. J Am Heart Assoc. 2019;8(10):e012290.

57. Kieliszek M, Lipinski B, Błażejak S. Application of sodium selenite in the prevention and treatment of cancers. Cells. 2017;6(4):39.

58. Sanmartín C, Plano D, Sharma AK, Palop JA. Selenium compounds, apoptosis and other types of cell death: An overview for cancer therapy. IJMS 2012;13(8):9649-72.

59. Mehdi Y, Hornick JL, Istasse L, Dufrasne I. Selenium in the environment, metabolism and involvement in body functions. Molecules. 2013;18(3):3292-311.

60. Natasha, Shahid M, Niazi NK, et al. A critical review of selenium biogeochemical behavior in soil-plant system with an inference to human health. Environ Poll. 2018;234:915-34.

61. Kieliszek M. Selenium - fascinating microelement, properties and sources in food. Molecules. 2019;24(7). [https://www.ncbi.nlm.nih.gov/pmc/articles/PMC6480557.]

62. He Y, Xiang Y, Zhou Y, et al. Selenium contamination, consequences and remediation techniques in water and soils: A review. Environ Res. 2018;164:288-301.

63. Lynam MM, Dvonch JT, Hall NL, et al. Spatial patterns in wet and dry deposition of atmospheric mercury and trace elements in central Illinois, USA. Environ Sci Pollut Res Int. 2014;21(6):4032-43.

64. Burk RF, Hill KE. Regulation of selenium metabolism and transport. Annu Rev Nutr. 2015;35:109-34.

65. Constantinescu-Aruxandei D, Frîncu R, Capră L, Oancea F. Selenium analysis and speciation in dietary supplements based on next-generation selenium ingredients. Nutrients. 2018;10(10):1466.

66. Groth S, Budke C, Neugart S, et al. Influence of a selenium biofortification on antioxidant properties and phenolic compounds of apples (Malus domestica). Antioxidants. 2020;9(2):187

67. Schomburg L. The other view: the trace element selenium as a micronutrient in thyroid disease, diabetes, and beyond. Hormones (Athens). 2020;19(1):15-24

68. Rayman MP. Selenium intake, status, and health: a complex relationship. Hormones (Athens). 2020;19(1):9-14.

69. Wojtasik A, Jarosz M, Stoś K. Składniki mineralne. In: M. Jarosz (ed.) Normy żywienia dla populacji Polski. Warszawa: Instytut Żywności i Żywienia; 2017. p. 203-37.

\section{Corresponding author}

Prof. dr hab. Barbara Nieradko-Iwanicka

Chair and Department of Hygiene, Medical University of Lublin

11 Radziwiłłowska St., 20-080 Lublin

E-mail: barbara.nieradko-iwanicka@umlub.pl 